\title{
Standard Score Analysis: Interpretive software for aiding mental retardation identification
}

\author{
LARRY D. EVANS \\ University of Arkansas for Medical Sciences, Little Rock, Arkansas
}

\begin{abstract}
Federal and most state guidelines mandate mental retardation identification based on intelligence and adaptive behavior scores. This paper describes Standard Score Analysis: Mental Retardation (SSA: MR), a computer program that interprets IQ and adaptive behavior scores according to cutoff values, measurement error, and IQ-adaptive behavior correlations. SSA: MR yields probabilities that each score is below its respective cutoff, and that IQ and adaptive behavior scores are simultaneously below cutoffs.
\end{abstract}

Until the early 1970 s mental retardation (MR) determination was based primarily on results of intelligence tests. In the 70s, however, concern developed for a more ecological assessment of mental functioning, and results from adaptive behavior measures were added to diagnostic criteria. At the time, adaptive behavior was defined by the American Association of Mental Deficiency as the individual's ability to behave in a manner required to meet environmental demands (Grossman, 1973). Sattler (1988) has characterized adaptive behavior primarily as maturation during preschool years, academic competence during school-age years, and social and economic independence in adulthood. Today, federal guidelines for the identification of students with educational disabilities require adaptive behavior and intelligence assessment for MR determination (Individuals with Disabilities Educational Act, Public Law 94-142, 300.5[3].).

The federal guidelines for MR identification do not specify cutoff indices for IQ and adaptive behavior scores, but do require that students demonstrate subaverage general intelligence and concurrent deficits in adaptive behavior. Subaverage intelligence may be defined by test publishers, state education departments, or local school districts. State values may be adjusted according to differences between mean IQ scores from state and national samples (e.g., Iowa Department of Public Instruction, 1981). Deficits in adaptive behavior are not frequently defined by states (Patrick \& Reschley, 1982), but by test publishers or local school districts. Generally, publishers of intelligence and adaptive behavior measures define overall scores less than or equal to two standard deviations below the mean as consistent with MR.

SSA: MR is available from WtL Publishing, 6929 JFK Blvd., Suite 20-120, North Little Rock, AR 72116. Address correspondence to L. D. Evans, 1612 Maryland St., Little Rock, AR 72202.

Note: The author has a direct financial interest in the software described in this paper.-Editor

\section{Purpose of SSA: MR}

Reliable determination of MR requires that both IQ and adaptive behavior scores fall at or below predefined cutoff values. Such cutoff values may be derived from multivariate classification based on the profile of adaptive behavior and intelligence scores (Resavy, 1993), or from bivariate classification based only on the overall adaptive behavior and intelligence scores. Because of the variability in the number and type of domains assessed by intelligence and adaptive behavior measures, as well as the manner in which federal MR identification guidelines are stated, multivariate results might vary too greatly according to the specific instruments used and identify score patterns as being severe on the basis of consistently low, but not deficit, scores. At present, bivariate classification procedures appear to offer greater reliability in classification decisions.

Framing MR identification as a bivariate classification procedure (i.e., the presence of subaverage overall intelligence existing concurrently with overall deficits in adaptive behavior) introduces statistical procedures that cannot readily be performed by hand calculation. The use of printed tables would require tables for each combination of intelligence and adaptive behavior measures at each age level. The purpose of Standard Score Analysis: Mental Retardation (SSA: MR) is to provide bivariate classification results to aid MR identification in a flexible and timely manner. The primary outcome of SSA: MR is a probability statement that both IQ and adaptive behavior scores are simultaneously at or below their respective cutoff values.

\section{Function of SSA: MR}

SSA: MR initially examines the probability that the overall IQ and adaptive behavior true scores are individually at or below their respective cutoff indices indicating subaverage (IQ) and deficit (adaptive behavior) performance. (Cutoff values are entered by the user and need not be the same value for intelligence and adaptive behavior scores.) Probability values result from consideration for measurement error, which is required when one 
is classifying continuous scores into dichotomous categories (Braden \& Algina, 1989). Each score's standard error of measurement $\left(S E_{\mathrm{m}}\right)$ is calculated from the entered reliability coefficients. Because measurement error is symmetric about estimated true scores and not obtained scores, estimated true scores are derived for both obtained scores. Conversion of obtained scores into estimated true scores requires a comparable transformation of cutoff indices into estimated true cutoff values. Each estimated true score's distance from its estimated true cutoff value is then measured with respect to its $S E_{\mathrm{m}}$. The result is evaluated according to univariate normal curve values to determine the probability that each true score is at or below its cutoff.

For bivariate MR classification, the critical issue is the likelihood that both IQ and adaptive behavior scores fall simultaneously at or below their respective cutoff indices (i.e., concurrent subaverage intelligence and adaptive behavior deficits). The probability that each score is individually at or below its cutoff is significant only as an indication of the upper limit of the simultaneous probability value. Calculation of the simultaneous probability value requires the user to enter the correlation coefficient between IQ and adaptive behavior scores. SSA: MR uses this coefficient with the test means and standard deviations to determine the bivariate distribution of IQ and adaptive behavior scores. The student's bivariate estimated true score is placed within the distribution, as is the bivariate estimated true cutoff value. SSA: MR then simulates a bivariate distribution of measurement error about the student's score. This distribution is based on the $S E_{\mathrm{m}} \mathrm{S}$ and correlation between scores. The simulation may include 100-20,000 scores (user selected). The percentage of scores from this distribution falling on or below the cutoff value represents the likelihood that both scores are simultaneously in the MR range.

\section{SSA: MR Features}

SSA: MR 1.1 includes the following features:

1. It is available for IBM and compatible computers.

2. Test standard deviations, reliabilities, correlations, and means can be stored in easily updated and modified computer files.

3. Cutoff values for MR can be selected and saved. Intelligence and adaptive behavior cutoffs can be the same or separate values.

4. The number of cases simulated for joint probability calculations can be selected. Values from between 100 and 20,000 cases are permissible. The number of cases selected affects the accuracy of results and SSA: MR run time.

5. Required test data can be loaded from files, a single set of saved data, or manually. Printed and screen outputs are available so that one may view results.

\section{SSA: MR Results}

Figure 1 shows the printed output of SSA: MR. The initial lines present the identifying data, option settings, and test data used to calculate the results. Because the student received the score of 65 on both the IQ and the adaptive measures, and because both scores have a reliability of .90 , the probability that each score is at or below its cutoff of 70 (i.e., $-2.00 \mathrm{SD}$ ) is $84.13 \%$. More importantly, the probability that the scores are simultaneously below their cutoffs is $74.89 \%$. Thus, the chances are nearly 3 out of 4 that the student's true scores are in the defined MR range. If the evaluator must make a recommendation solely on the basis of the test data, the student would appear more likely to have scores in the MR range than in the non-MR range.

If the scores shown in Figure 1 were more reliable (e.g., .95 for each), SSA: MR would indicate that the probability that both scores were simultaneously at or below their cutoffs would increase to $87.38 \%$. If the reliabilities should remain at .90 , but the correlation should increase to .65 , this value would become $76.44 \%$.

SSA: MR may also be useful when a practitioner must render an opinion about a student's MR level. For example, if scores $>40$ yet $\leq 55$ are considered to indicate moderate MR and a student has an IQ of 50 and an overall adaptive behavior score of 56, SSA: MR can be used to determine the probability that the scores are more likely in the moderate or mild MR range. To accomplish this, the Option Settings of SSA: MR can be input as -3.00 (for measures with a 15-point SD). The final probability value on the printout is the likelihood that scores are $\leq \mathbf{5 5}$ and not in the mild MR range.

\section{SSA: MR Limitations}

Reschley (1985) has argued that cutoff scores are not indicated for the adaptive behavior assessment of MR determination. Because no single instrument can assess all relevant adaptive behavior domains (Sattler, 1988), Reschley recommends multiple scores and measures. Unfortunately, no method is offered to address the considerable multivariate classification issues that arise, or to determine whether deficits in a few domains are necessarily characteristic of MR populations.

Bias in adaptive behavior or intelligence assessment may adversely affect scores interpreted by SSA: MR. Adaptive behavior measures appear to be more susceptible to bias, owing to greater variation in assessment than in intelligence measures. Issues such as assessment of home versus school behavior, choice of informant, scoring of items according to what the child actually does versus can do may introduce bias for some groups of students and thereby affect MR determination (Evans \& BradleyJohnson, 1988).

Some states (e.g., Michigan) have added academic achievement criteria to MR identification procedures. Similarly, recent guidelines of the American Association on Mental Retardation (1992) require "limitations" in two or more areas of adaptive behavior existing concurrently with subaverage IQ. SSA: MR offers limited interpretive data in cases with more than two scores. However, 
STANDARD SCORE ANALYSIS 1.1

IDENTIFYING DATA

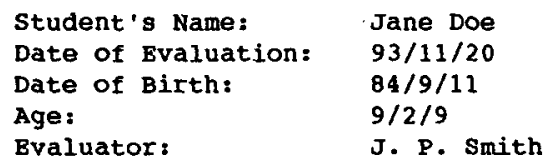

End Printout Serial Number I3SSA0004

Figure 1. Example of the SSA: MR printed output.

SSA:MR can be used with the two scores that exceed or are closest to exceeding their cutoffs by the greatest amount. The result will be a probability that serves as an upper bound for the multivariate probability that all variables fall at or below cutoffs.

\section{Conclusions}

As classification decisions become increasingly based on multiple data sources, practitioners are challenged to draw conclusions that are statistically sound. Computer programs that go beyond interpretation of single scores and offer options for considering data from other measures will be needed. Standard Score Analysis: Mental Retardation provides interpretive information for IQ and adaptive behavior scores by calculating the joint probability that scores fall at or below cutoff indices. Because the majority of classification decisions go beyond a bivariate model, future software will need to address multivariate classification issues such as multiple cutoffs, score weights, multiple comparisons, the correlation matrix, and measurement error.

\section{REFERENCES}

American Association on Mental Retardation (1992). Mental retardation: Definition, classification, and systems of support (9th ed.). Washington, DC: Author.

Braden, J. P., \& Algina, J. (1989). A method for determining the probability of special education eligibility. Journal of School Psychology, 27, 5-13.

Evans, L. D., \& Bradley-Johnson, S. (1988). A review of recently developed measures of adaptive behavior. Psychology in the Schools, 25, 276-287.

GrossmaN, H. J. (1973). Manual on terminology and classification in mental retardation (1973 revision). Baltimore: Garamond/Pridemark.

Iowa Department of Public Instruction (1981). The identification of pupils with learning disabilities. Des Moines, IA: Author.

PATrick, J., \& Reschley, D. (1982). Relationship of state education criteria and demographic variables to school-system prevalence of mental retardation. American Journal of Mental Deficiency, 86, 351-360.

Resavy, C. P. (1993, April). Mental retardation: A cluster analytic approach to classification. Paper presented at the convention of the National Association of School Psychologists, Washington, DC.

RESCHLEY, D. (1985). Best practices: Adaptive behavior. In A. Thomas \& J. Grimes (Eds.), Best practices in school psychology (pp. 353368). Kent, OH: National Association of School Psychologists.

SatTler, J. M. (1988). Assessment of children (3rd ed.). San Diego: Author. 